\title{
TRAVELING WAVE SOLUTIONS FOR INTERFACES ARISING FROM PHASE BOUNDARIES BASED ON A PHASE FIELD MODEL
}

\author{
By \\ J. W. WILDER \\ West Virginia University, Morgantown, West Virginia
}

\begin{abstract}
This work presents an analysis of the traveling wave solutions resulting from the phase field model which has been proposed for solidification. It is shown that solutions only exist for very restricted values of the parameters involved and these values are investigated. The basic nature of the traveling wave solutions resulting from this model are also discussed.
\end{abstract}

Introduction. Most of the work which has been done in the area of solidification theory has been based on the Stefan problem [9] which considers the incorporation of heat diffusion and the latent heat of a material into the problem of solidification of a homogeneous material. While the resulting equations incorporate much of the physics of the problem, they do have certain limitations. For example, the interface is considered to be infinitesimally thin, and there is no way to investigate the connection between lattice concepts such as correlation lengths and lattice anisotropy with the appropriate properties of the continuum model.

One proposed approach to eliminate these limitations is to consider a set of Model A phase field equations which result from the consideration of an appropriate Landau-Ginzberg free energy functional [2]. Caginalp and Fife have presented various aspects of the formulation and analysis of these phase field equations [3, 4]. They found that the phase field formulation allowed for the use of higher-order equations which revealed more detail in the structure of the interface [4], and that it incorporated surface tension, anisotropy, curvature, and dynamics of the interface as well as allowing for supercooling of the liquid [3]. Caginalp and Fife [5] as well as Caginalp and Fife [5] as well as Caginalp [1] have examined the resulting system and have presented various existence and uniqueness theorems. Caginalp and McLeod [7] have examined the steady state solutions of this system in the spherically symmetric case resulting in a Gibbs-Thompson relation relating the temperature at the interface to the curvature of the interface and the surface tension. Caginalp and Fife [6] have presented similar Gibbs-Thompson relations for the transient system of equations through the use of matched inner and outer expansions.

Received May 30, 1990.

(C)1991 Brown University 
Lin [8] has presented some numerical results based on a system of phase field equations. However, they are slightly different from those used by Caginalp et al. These differences will be pointed out in the next section when we state the problem in a more formal manner. In this paper Lin states that the solutions obtained for a particular case are on the order of $1 \%$ different from the solutions to the Stefan problem, and Lin therefore proposes that this is a good indication of the veracity of this model. The results of this work, however, seem to contradict this statement, at least in the case of traveling wave solutions. This will be discussed in more detail in the Conclusions section of this work.

As stated by Caginalp and Fife [3], for the transient (or nonequilibrium) case the validity of their formal analysis is contingent upon the existence of appropriate traveling wave solutions. Since this represents a case which is somewhat simpler to solve than the full problem and can thereby allow for at least a partial evaluation of the effectiveness of this phase field model, in this work we shall be concerned with finding these traveling wave solutions and examining what they can tell us about the model.

Problem formulation. As mentioned above, the problem which shall be considered here involves the phase field equations suggested by Caginalp et al. [1-7] to model solidification problems. The system of equations is

$$
\begin{gathered}
\tau \phi_{t^{\prime}}=\xi^{2} \phi_{x^{\prime} x^{\prime}}+\tilde{f}(\phi)+\sigma T, \\
T_{t^{\prime}}+\frac{l}{2} \phi_{t^{\prime}}=\kappa T_{x^{\prime} x^{\prime}},
\end{gathered}
$$

where $\tau$ is a relaxation time, $\xi$ is a correlation length (which measures the strength of the bonding in the material [2], the finite nature of which results in nonzero values for surface tension and interface width [4]), $\sigma$ is an entropy coefficient, $l$ the ratio of latent and specific heats, $\kappa$ the thermal diffusivity, $\phi$ an order parameter, $T$ the temperature relative to the melting temperature of the material and $\widetilde{f}(\phi)$ a function related to the free energy functional for the system.

In the above mentioned work by Lin, " $\sigma$ " is replaced by $2 \rho C / L$, where $\rho$ is the density of the material, $C$ is the specific heat and $L$ the latent heat. This would not have any effect on the results of this work since it would merely require that in the nondimensionalization outlined below $\sigma$ be replaced by $2 / l$. Since (1) is the more general form of the model, we shall be concerned with it.

We see that this system will allow constant solutions for $\phi$ and $T$ iff

$$
\widetilde{f}(\phi)+\sigma T=0 .
$$

This implies, as discussed by Caginalp and Fife [6], that $\tilde{f}(\phi)$ is bounded by $\pm \sigma T^{*}$ where $T^{*}$ is some maximum amount of supercooling which is allowable if we expect to have constant solutions in some region of the $\left(x^{\prime}, t^{\prime}\right)$ space. In this work we shall be considering a prototype double-well potential to model the phase transition, which leads to $\tilde{f}(\phi)$ being of the form $\tilde{f}(\phi)=\frac{1}{2}\left(\phi-\phi^{3}\right)$. Since we are here only interested in values of $\phi$ between +1 and -1 [2], we see that the maximum and minimum occur at $\phi$ values of $1 / \sqrt{3}$ and $-1 / \sqrt{3}$, respectively. The magnitude of 
$\tilde{f}(\phi)$ for these values of $\phi$ is $1 /(3 \sqrt{3})$. This implies that $T^{*}=1 /(3 \sqrt{3} \sigma)$. This then is some characteristic temperature which, as stated above, corresponds to the maximal amount of supercooling which can be used and obtain constant solutions.

The problem can be nondimensionalized by the following choice of dimensionless parameters:

$$
\begin{gathered}
x=\frac{x^{\prime}}{\xi}, \quad t=\frac{t^{\prime} \kappa}{\xi^{2}}, \quad u=T \sigma, \\
\alpha=\frac{\tau \kappa}{\xi^{2}}, \quad \lambda=\frac{l \sigma}{2} .
\end{gathered}
$$

This leads to

$$
\begin{gathered}
u_{t}+\lambda \phi_{t}=u_{x x}, \\
\alpha \phi_{t}=\phi_{x x}+\tilde{f}(\phi)+u,
\end{gathered}
$$

where $\tilde{f}(\phi)=\frac{1}{2}\left(\phi-\phi^{3}\right)$, as used by Caginalp and Fife. Other choices for $\tilde{f}(\phi)$ are possible, however, this one corresponds to a commonly used prototype double-well potential of the form $\left(\phi^{2}-1\right)^{2}$.

If we examine the definition of $u$ given above, it is seen that under conditions where the largest amount of supercooling is applied, then $u=-\sigma T^{*}=-1 /(3 \sqrt{3})$. This gives a bound on $u$, namely that $|u| \leq 1 /(3 \sqrt{3})$. This condition must be remembered when formulating boundary conditions for the problem. We should note that this does not imply that the boundary condition should be $-1 /(3 \sqrt{3})$ for the following reason: if we consider solutions which become constant as the boundary is approached (for example one boundary could be at $x=+\infty$ or $x=-\infty$ ), then we would require

$$
\tilde{f}\left(\phi_{b}\right)+\sigma T_{b}=0,
$$

where " $b$ " refers to the value at the boundary. Thus,

$$
u_{b}=-\tilde{f}\left(\phi_{b}\right) \text {, }
$$

which is not $\pm 1 /(3 \sqrt{3})$ unless the boundary condition on $\phi$ is picked so that $\phi_{b}=$ $\pm 1 / \sqrt{3}$. This demonstrates that $T^{*}$ is not the value of the supercooling at the boundary, but is the maximum value one could pick for a boundary condition on the temperature and still allow for constant solutions near the boundary. This will be important since we wish to consider solutions which are constant far behind and far in front of a wavefront traveling with a constant speed.

If we look for traveling wave solutions where $X=x-c t$ and $t^{*}=t$ we find the system of equations to take the form

$$
\begin{gathered}
-c u_{X}+u_{t^{*}}+\lambda\left(-c \phi_{X}+\phi_{t^{*}}\right)=u_{X X}, \\
\alpha\left(-c \phi_{X}+\phi_{t^{*}}\right)=\phi_{X X}+f(\phi)+u .
\end{gathered}
$$

Examining these under the restriction that the front does not change shape with time 
(or does so very slowly), we have

$$
\begin{gathered}
u_{X X}+c u_{X}+c \lambda \phi_{X}=0 \\
\phi_{X X}+\frac{1}{2}\left(\phi-\phi^{3}\right)+\alpha c \phi_{X}+u=0 .
\end{gathered}
$$

Equation (4a) can be integrated once immediately to give

$$
u_{X}+c u+c \lambda \phi=\text { const }
$$

Since as $X \rightarrow-\infty, u_{X} \rightarrow 0, u \rightarrow u_{-\infty}$, and $\phi \rightarrow \phi_{-\infty}$ (which are known from boundary conditions), (4) becomes:

$$
\begin{aligned}
& u_{X}+c u+c \lambda \phi=c\left(u_{-\infty}+\lambda \phi_{-\infty}\right), \\
& \phi_{X X}+\frac{1}{2}\left(\phi-\phi^{3}\right)+\alpha c \phi_{X}+u=0 .
\end{aligned}
$$

The question as to what the appropriate boundary conditions are shall be discussed in the next section where the necessary choices become more obvious. In the following sections we shall first examine the behavior of these equations near the singular points of the system. This information will then be used to examine the full problem described by (5).

I. The singular points. In order to examine the singular points we will be working in an $(s, u, \phi)$ phase space, where $s=\phi_{X}$. In this notation, (5) becomes

$$
\begin{gathered}
\phi_{X}=s \\
s_{X}=-\frac{1}{2}\left(\phi-\phi^{3}\right)-\alpha c s-u, \\
u_{X}=-c\left(u-u_{-\infty}\right)-c \lambda\left(\phi-\phi_{-\infty}\right) .
\end{gathered}
$$

From (6) we see that there are singular points when

$$
\begin{gathered}
s=0, \\
\frac{1}{2}\left(\phi-\phi^{3}\right)+u=0, \\
\left(u-u_{-\infty}\right)+\lambda\left(\phi-\phi_{-\infty}\right)=0 .
\end{gathered}
$$

Since we now want to consider the problem to be fully infinite (i.e., running from $+\infty$ to $-\infty)$, as $X \rightarrow \pm \infty$ the solution must enter a singular point in order to remain bounded at infinity. Thus, (7) must be obeyed at $\pm \infty$, which allow us to determine the boundary conditions needed to solve the problem. Using (7) we find

$$
\begin{gathered}
s_{ \pm \infty}=0, \\
\frac{1}{2}\left(\phi_{ \pm \infty}-\phi_{ \pm \infty}^{3}\right)+u_{ \pm \infty}=0, \\
\left(u_{ \pm \infty}-u_{-\infty}\right)+\lambda\left(\phi_{ \pm \infty}-\phi_{-\infty}\right)=0 .
\end{gathered}
$$

We see that (8c) is satisfied for any choice of $u_{-\infty}$ and $\phi_{-\infty}$, leaving (8b) to determine the relationship between $u_{-\infty}$ and $\phi_{-\infty}$. If the case of a solidification front 
is considered, $X \rightarrow-\infty$ corresponds to the solid region. In this region the temperature is the fusion temperature, which in nondimensional form implies $u_{-\infty}=0$. Using this in (8b) gives $\phi_{-\infty}=-1$. This is as we might expect from the form of the double-well potential given above. For this type of well $\phi= \pm 1$ corresponds to the most thermodynamically stable states for the material (with negative indicating a solid). Thus, for boundary conditions at $-\infty$ we have

$$
\begin{aligned}
& s_{-\infty}=0, \\
& u_{-\infty}=0, \\
& \phi_{-\infty}=-1 .
\end{aligned}
$$

Using these values to find the equations for the boundary conditions at $+\infty$ leads to

$$
\begin{gathered}
u_{+\infty}+\lambda\left(\phi_{+\infty}+1\right)=0, \\
\frac{1}{2}\left(\phi_{+\infty}-\phi_{+\infty}^{3}\right)+u_{+\infty}=0 .
\end{gathered}
$$

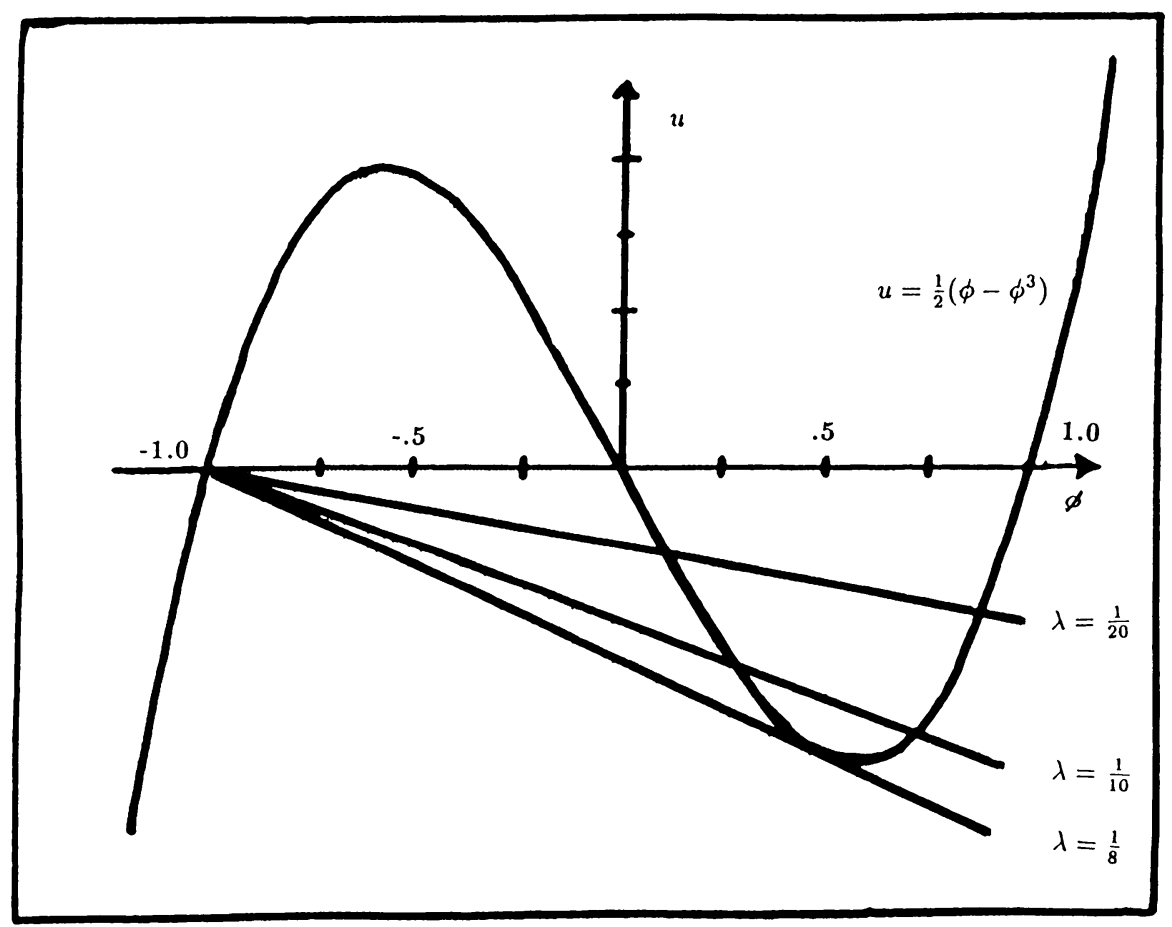

Fig. 1. The singular points of the equations. The intersections represent the singular points

These curves are plotted in Fig. 1. From Fig. 1 it is observed that the value of $\lambda$ determines whether there will be one, two, or three real singular points, since it determines the slope of the straight line coming out of the point $\phi_{-\infty}=-1$, 
$u_{-\infty}=0$. The factored form of the equation for $\phi_{+\infty}$ obtained from (10) is

$$
\left(\phi_{+\infty}+1\right)\left(\phi_{+\infty}-\left[\frac{1}{2}+\sqrt{\frac{1}{4}-2 \lambda}\right]\right)\left(\phi_{+\infty}-\left[\frac{1}{2}-\sqrt{\frac{1}{4}-2 \lambda}\right]\right)=0 .
$$

From (10c) it is obvious that when $\lambda$ is greater than $\frac{1}{8}$ two of the roots will be complex. Since a complex value of $\phi_{-\infty}$ implies a complex value of $u_{+\infty}$ (which is nonphysical), for $\lambda$ greater than $\frac{1}{8}$ the only possibility for a singular point as $X \rightarrow+\infty$ is $\left(\phi_{+\infty}=-1, u_{+\infty}=0\right)$, which is the boundary condition at $-\infty$. This implies we would have a solid at both $X= \pm \infty$, which is a case we are not interested in here. We should also note that based on physical grounds, only cases where the latent heat is greater than zero (i.e., $\lambda \geq 0$ ) will be considered. These remarks allow the following general conclusion: for systems where the latent heat and heat capacity are such that $\lambda$ is greater than this critical value of $\frac{1}{8}$, no traveling wave solutions of the form we are considering here are possible. For values of $\lambda$ less than this there are one or two singular points which may allow such solutions. In the case where such solutions are possible, (10a) shows that they will require the liquid to be supercooled since $\lambda$ and $\phi_{+\infty}$ are both greater than zero. The implications of these restrictions will be discussed more fully in the last section of this work.

II. The nature of the singular points. The local analysis of the equations near the singular points must now be done to determine the nature of the solutions in these regions. We here consider:

$$
\begin{gathered}
\phi=\phi_{s}+\varphi, \\
u=u_{s}+\omega, \\
s=s_{s}+\tau,
\end{gathered}
$$

where the subscript " $s$ " refers to the value at the singular point, and the added function is some small correction. Using (11) in (6) and considering (9b) leads to

$$
\begin{gathered}
\varphi_{X}=\tau, \\
\omega_{X}=-c \omega-c \lambda \varphi, \\
\tau_{X}=-\omega-\alpha c \tau-\frac{1}{2}\left(1-3 \phi_{s}^{2}\right) \varphi,
\end{gathered}
$$

where the equations have been linearized with respect to the small corrections $\varphi$, $\omega$, and $\tau$.

The solutions to this system are found to be

$$
\begin{gathered}
\varphi=\Phi_{1} e^{c \rho_{1} X}+\Phi_{2} e^{c \rho_{2} X}+\Phi_{3} e^{c \rho_{3} X}, \\
\omega=W_{1} e^{c \rho_{1} X}+W_{2} e^{c \rho_{2} X}+W_{3} e^{c \rho_{3} X}, \\
\tau=S_{1} e^{c \rho_{1} X}+S_{2} e^{c \rho_{2} X}+S_{3} e^{c \rho_{3} X},
\end{gathered}
$$

where the $\rho_{i}$ are the three roots of the equation

$$
\rho^{3}+(1+\alpha) \rho^{2}+(\alpha+\Psi) \rho+(\Psi-\Lambda)=0,
$$


with

$$
\Psi=\frac{1-3 \phi_{s}^{2}}{2 c^{2}} ; \quad \Lambda=\frac{\lambda}{c^{2}} .
$$

The relationships between the coefficients of (13) are given by

$$
\begin{gathered}
S_{i}=c \rho_{i} \Phi_{i}, \\
W_{i}=-\left(c^{2} \rho_{i}\left(\rho_{i}+\alpha\right)+\frac{\left(1-3 \phi_{s}^{2}\right)}{2}\right) \Phi_{i},
\end{gathered}
$$

for $i=1,2$, and 3 . One can now, for any choice of $c, \lambda$, and $\alpha$ examine the nature of the solutions near the singular points. As an example we shall consider the case when $c^{2}=0.1, \lambda=0.1$, and $\alpha=1$. In this case there are three singular points at $(s, \phi, u)$ coordinates of $(0,-1,0),(0,0.276,-0.127)$ and $(0,0.724,-0.172)$. For the singular points at $(0,-1,0)$ and $(0,0.724,-0.172)$ the roots of $(14)$ are all real (with one being greater than zero and the other two less than zero), meaning that these two are saddle points. On the other hand, (14) has one real (negative) root and two complex roots when the singular point at $(0,0.276,0.127)$ is considered, showing that this singular point is spiral in nature. Due to the nature of the saddle points, if we wish to consider solutions where $s$ remains positive and $\phi$ is increasing as $X$ increases, then for this choice of $(c, \lambda, \alpha)$ there is a unique trajectory which comes out of $\mathscr{S}_{-}$(the singular point with the most negative $\phi$ value) and which goes into $\mathscr{S}_{+}$(the singular point with the most positive $\phi$ value). Under what conditions these singular points can be connected will be discussed in the next section. We should also note that for some choice of $(c, \lambda, \alpha)$ there are also trajectories which enter $\mathscr{S}_{0}$ (the singular point located between $\mathscr{S}_{-}$and $\mathscr{S}_{+}$). As we shall see shortly, the choice of these parameters is very important to the nature of the singular points, and thereby the overall solution. We must therefore examine the nature of the singular points in more depth.

To investigate what happens to the singular points as $c, \lambda$, and $\alpha$ are varied, the roots of (14) must be examined for each of the singular points. For $\mathscr{S}_{-}$it is found that (14) always has one real, positive root, while the other two roots can be either real (and negative) or complex (with negative real parts). Figure 2 shows the regions where the roots are complex. Interior to this volume the singular point is spiral in nature, while outside of this region it is a saddle point. In the region where we have a saddle point there is a unique trajectory which comes out of the singular point and is of the appropriate nature (i.e., having $s>0$ and $u<0$ ). All of the other trajectories do not originate at the singular point and so will not be bounded as $X \rightarrow-\infty$. Where the singular point is spiral in nature, there also exists a unique trajectory which comes out of the singular point without any oscillations in $u$ and $\phi$. All other trajectories lead to solutions for $\phi$ and $u$ which have a damped oscillatory nature when they are examined as functions of $X$. We wish to consider here only those solutions leaving $\mathscr{S}_{-}$which are monotonic in nature near the singular point, since these would appear to correspond to the most physically relevant ones in this case where we are considering the solid to be in its most thermodynamically stable state. 


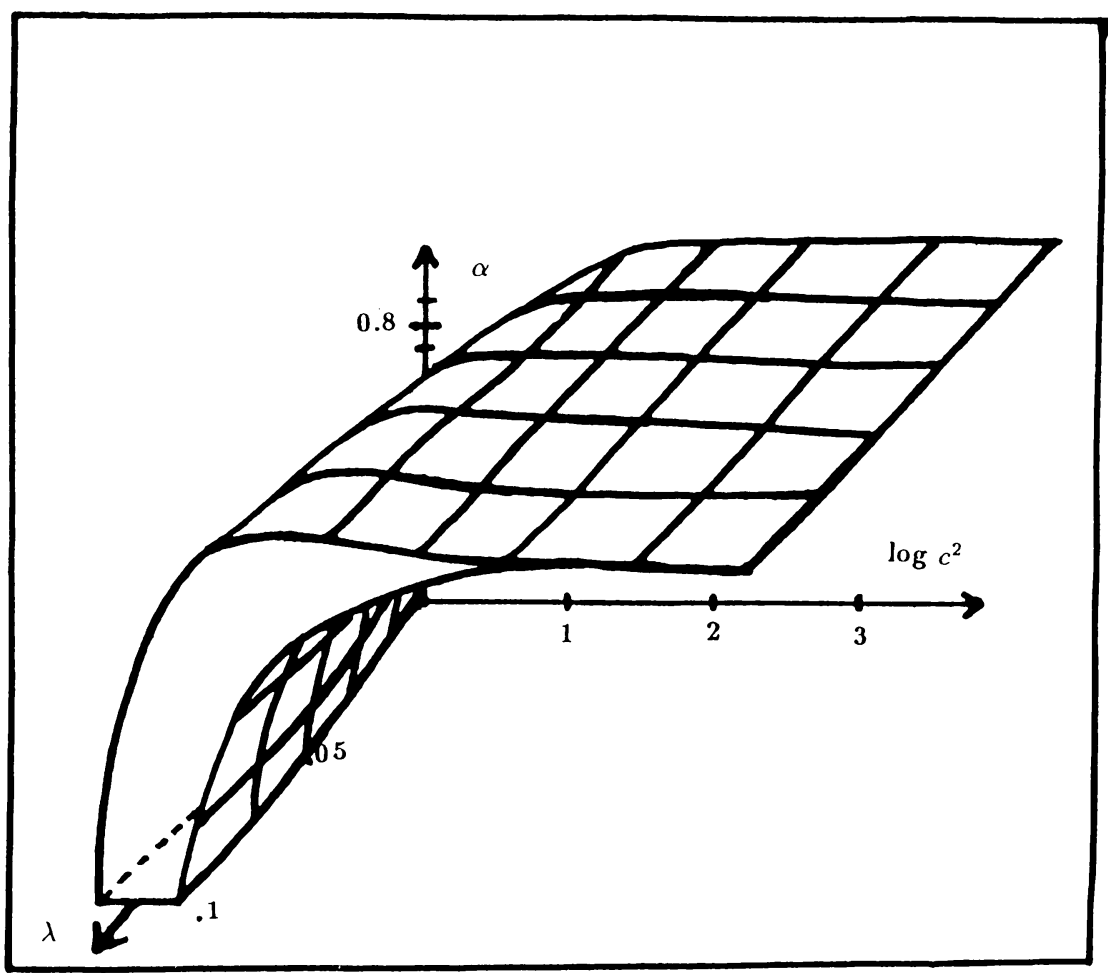

FIG. 2. The nature of singular point $\mathscr{S}_{-}$. Inside the surfaces the singular point is a vortex. Outside it is a saddle point.

Examining $\mathscr{S}_{0}$ in a similar manner leads to Fig. 3, where once again the region interior to the surfaces defines the region where the singular point is spiral in nature. Outside this volume the singular point is a node. In this case we find that (14) always has one real, negative root and two roots which may be either real (and negative) or complex (with negative real parts). Similar to what was found above, we see that this singular point always has a unique trajectory which goes into the singular point without oscillations in $u$ or $\phi$.

Figure 4 shows the nature of $\mathscr{S}_{+}$. Interior to the surfaces shown here the singular point is spiral in nature, while outside of them it is a saddle point. Here it is found that (14) always has one real, positive root and two which may be either real (and negative) or complex (with negative real parts). If we examine what this implies for the behavior of $\phi$ near $\mathscr{S}_{+}$we find that inside the surfaces $\phi$ is of the form

$$
\phi \sim A e^{\rho_{1} X}+B e^{\left(\rho_{2 r}+i \rho_{21}\right) X}+C e^{\left(\rho_{2 r}-i \rho_{21}\right) X},
$$

while outside of this region the trajectories are of the form

$$
\phi \sim A e^{\rho_{1} X}+B e^{\rho_{2} X}+C e^{\rho_{3} X},
$$

where $\rho_{1}>0$ and $\rho_{2 r}, \rho_{2}$, and $\rho_{3}$ are all negative. If the trajectories are to enter the singular point as $X \rightarrow \infty$ then in either case $A$ must be zero. When the singular point is a saddle point this leads to trajectories with simple exponential decay. When 


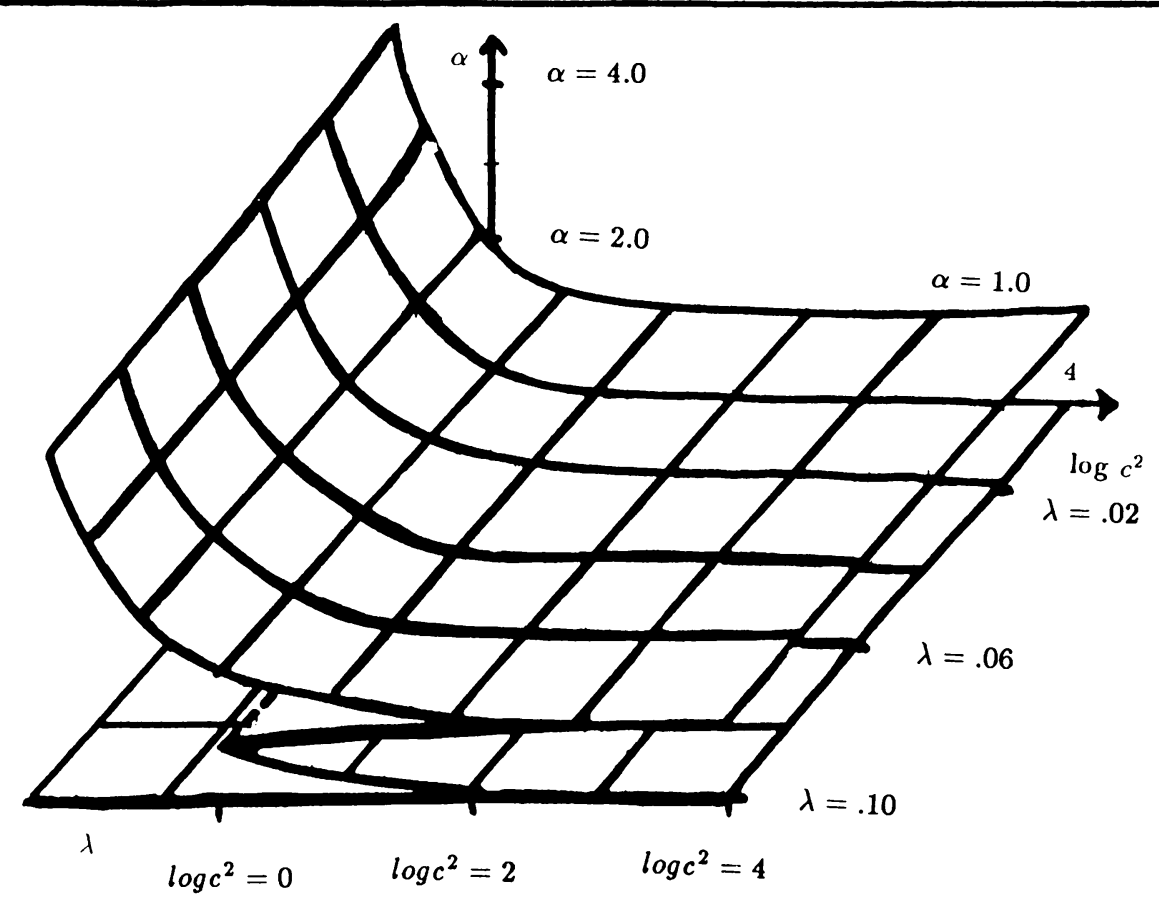

FIG. 3. The nature of singular point $\mathscr{S}_{0}$. Inside the surfaces the singular point is a vortex. Outside it is a saddle point.

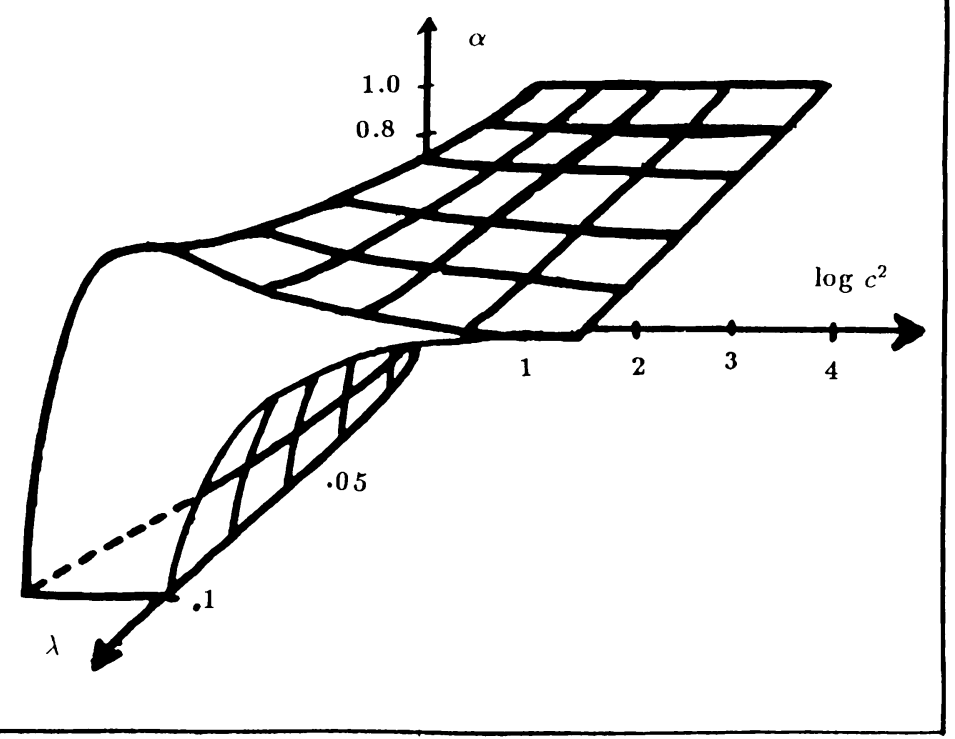

FIg. 4. The nature of singular point $\mathscr{S}_{+}$. Inside the surfaces the singular point is a vortex. Outside it is a saddle point. 
it is a spiral they have a damped oscillatory nature. In either case, however, it is plain from the above local solutions that there will always exist a unique solution which enters the singular point. The solutions which are oscillatory in the "liquid" region may correspond to the experimental observation of so-called "mushy zones" in which the state of the material alternates between solid-like and liquid-like forms. These oscillations near $\mathscr{S}_{0}$ and $\mathscr{S}_{+}$may not then be unphysical. This shall be discussed more fully later.

III. Connecting the singular points. In this section the solution to (7) in the whole $(s, \phi, u)$ space will be examined. To do this, we will use a fourth-order Runge-Kutta method to solve the problem in the region away from the critical points. The local solution near the singular point will be used as $X \rightarrow-\infty$ as the initial conditions for the computations. The numerical method we shall use can be outlined in the following manner: the approximate solution of the initial-value problem,

$$
y^{\prime}=f(X, y), \quad a \leq X, \quad y(a)=\beta,
$$

can be obtained by considering discrete points in $X$ with spacing $h$ to give an approximation $\tilde{y}$ to $y$. The solution at each point is then found by performing the following steps:

Step 1.

$$
\begin{aligned}
& X_{0}=a ; \\
& \tilde{y}_{0}=\beta ;
\end{aligned}
$$

Step 2. (Do for $i=1$ to $\mathbf{N}$, where $\mathbf{N}$ is the desired number of steps)

$$
\begin{aligned}
& R_{1, i}=h f\left(X_{i-1}, \tilde{y}_{i-1}\right) ; \\
& R_{2, i}=h f\left(X_{i-1}+h / 2, \tilde{y}_{i-1}+R_{1, i} / 2\right) ; \\
& R_{3, i}=h f\left(X_{i-1}+h / 2, \tilde{y}_{i-1}+R_{2, i} / 2\right) ; \\
& R_{4, i}=h f\left(X_{i-1}+h, \tilde{y}_{i-1}+R_{3, i}\right) ;
\end{aligned}
$$

with

$$
\begin{aligned}
\tilde{y}_{i+1} & =\tilde{y}_{i}+\left(R_{1, i}+2 R_{2, i}+2 R_{3, i}+R_{4, i}\right) / 6 ; \\
X_{i+1} & =a+i h .
\end{aligned}
$$

In this way the solution at each point $i$ can be calculated.

In the case being considered here, the "initial" condition is at $X=-\infty$. In order to solve the problem numerically we must have an initial condition at a finite value of $X$, and so we need the results of the local analysis above. Examining the saddle point at $(0,-1,0)$ we find that there is a unique trajectory which comes out of the saddle point with a positive value of $s$ (which is necessary since we want $\phi$ to increase and $u$ to decrease as $X$ increases), and which is monotonic. This trajectory is described by

$$
\begin{gathered}
s=S_{+} e^{c \rho_{+} x}, \\
\phi=-1+\Phi_{+} e^{c \rho_{+} X}, \\
u=W_{+} e^{c \rho_{+} X},
\end{gathered}
$$


where the "+" subscript indicates values corresponding to the positive, real root of (14) found for $\mathscr{S}_{-}$.

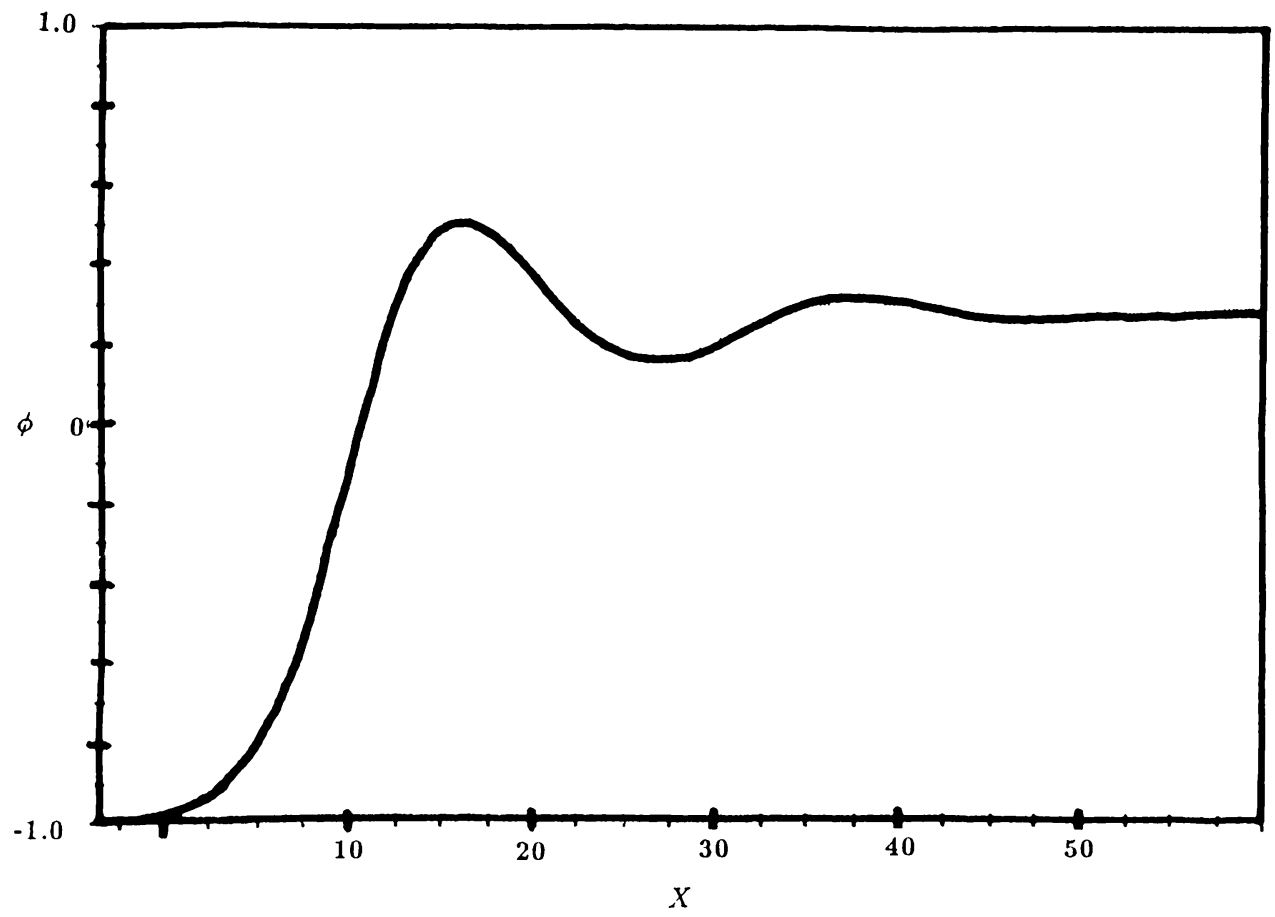

FIG. 5. $\phi$ vs. $X$ for $(c, \lambda, \alpha)=(\sqrt{.1}, 0.1,1.0)$.

Examining the solutions obtained for the case considered above (namely, $(c, \lambda, \alpha)$ $=(\sqrt{.1}, 0.1,1.0))$, we find the profiles shown in Figs. 5 and 6 for $\phi$ and $u$, respectively. From these figures it is seen that in this case the trajectory connects $\mathscr{S}_{-}$and $\mathscr{S}_{o}$ under conditions such that $\mathscr{S}_{o}$ is a spiral. This is an example of a trajectory which goes into $\mathscr{S}_{o}$ along a path which leads to the oscillatory solutions described above. Below we shall discuss this type of solution as compared with the solutions which enter the other saddle point.

We must now map out the $(c, \lambda, \alpha)$ values for which there are trajectories connecting the singular points. Doing so basically amounts to examining the possible trajectories coming out of $\mathscr{S}_{-}$and then extending them numerically to see where they go in the phase space. We shall first consider trajectories which end up in $\mathscr{S}_{+}$. The result is Fig. 7. In examining this figure we must first realize that interior to the region described above where $\mathscr{S}_{+}$is spiral in nature, there are no solutions which go into the singular point monotonically. There does exist, however, a solution which is oscillatory in this region. The surface representing these unique solutions which enter $\mathscr{S}_{+}$with oscillations is shown in Fig. 7. The surfaces shown in Fig. 7 which are on either side of the area where the solutions are oscillatory contain the points 


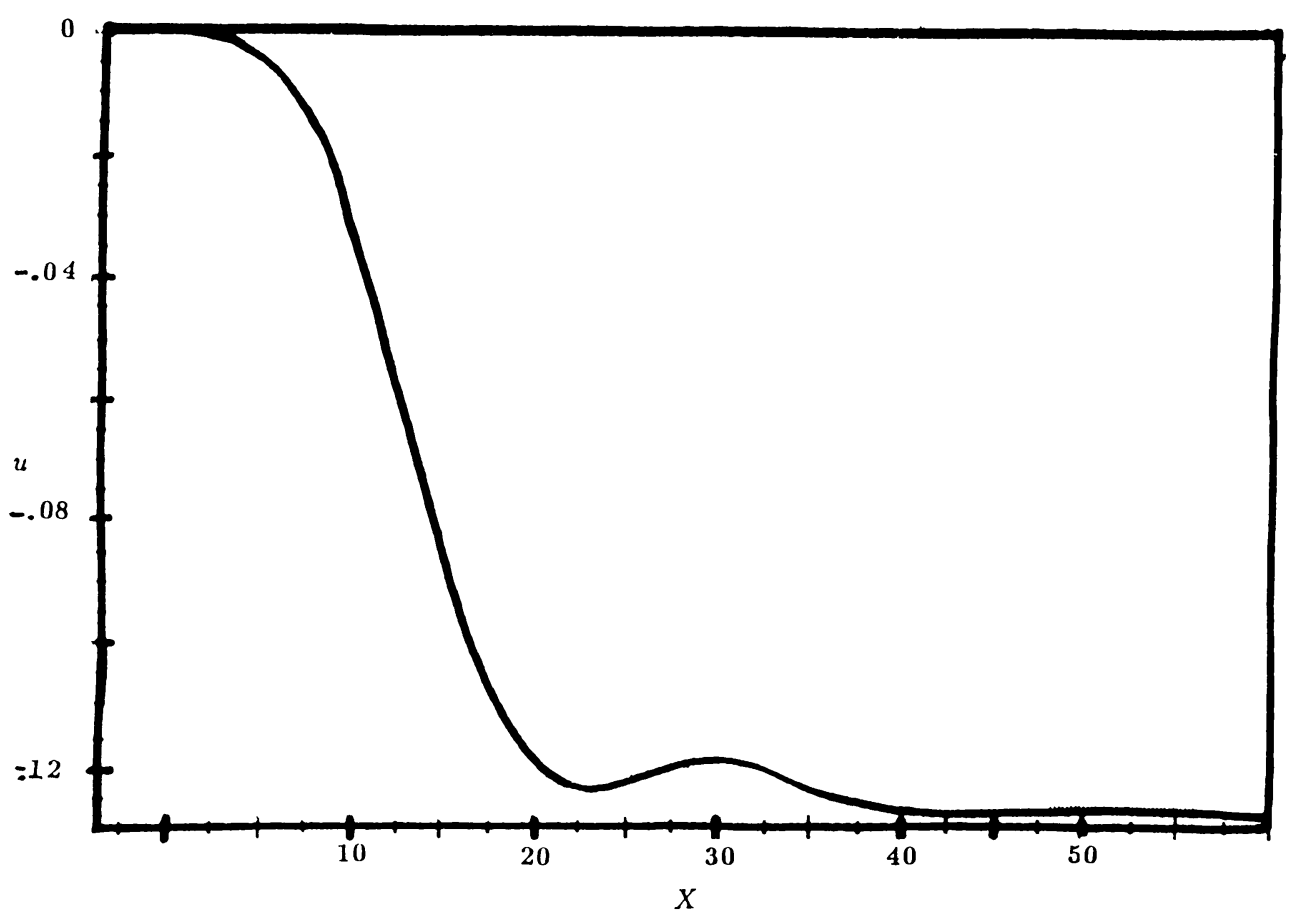

FIG. 6. $u$ vs. $X$ for $(c, \lambda, \alpha)=(\sqrt{.1}, 0.1,1.0)$.

for which the trajectories come out of $\mathscr{S}_{-}$and go into $\mathscr{S}_{+}$monotonically. Below these surfaces the solutions are not bounded as $X \rightarrow+\infty$, and so no solutions exist. Above them the trajectories go into $\mathscr{S}_{0}$.

If we now examine the trajectories which go into $\mathscr{S}_{0}$ we find that the surface which describes those which go into the singular point without oscillations includes and is an extension of the lower surface in Fig. 3, as shown in Fig. 8. Above this surface the trajectories go into $\mathscr{S}_{0}$ without any oscillations. Below this they go into the singular point with oscillations in $u$ and $\phi$ (except of course for those values discussed above for which the trajectories go into $\mathscr{S}_{+}$or which are not bounded as $X \rightarrow+\infty)$.

There are some general statements which can be made concerning the solutions as these parameters are varied. As $\alpha$ is increased the front becomes more spread out in $X$. This is as we would expect since $\alpha$ is related to a relaxation time (e.g., a measure of the time to reach equilibrium). As this is increased the system takes "longer" to undergo the phase transformation, resulting in a front which is more spread out. The front also spreads out as the speed $c$ is increased. We should note that we are here using the term front in its most broad sense (i.e., the region it takes for the transition to take place between $\phi=-1$ and the value at the singular point $\mathscr{S}_{+}$). This too seems to be what one would expect. As the speed of the wave increases, the area over which the transition is taking place increases because the phase transition takes a finite amount of time to occur. As the speed increases, the region where the solid 


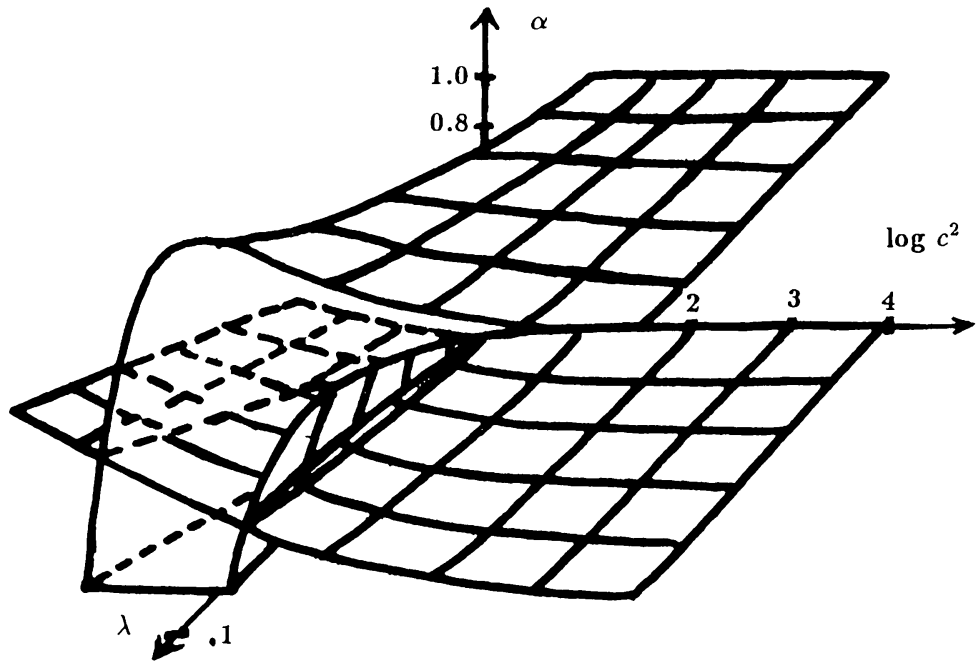

FIG. 7. Parameter values for trajectories which originate at $\mathscr{S}_{-}$and terminate at $\mathscr{S}_{+}$.

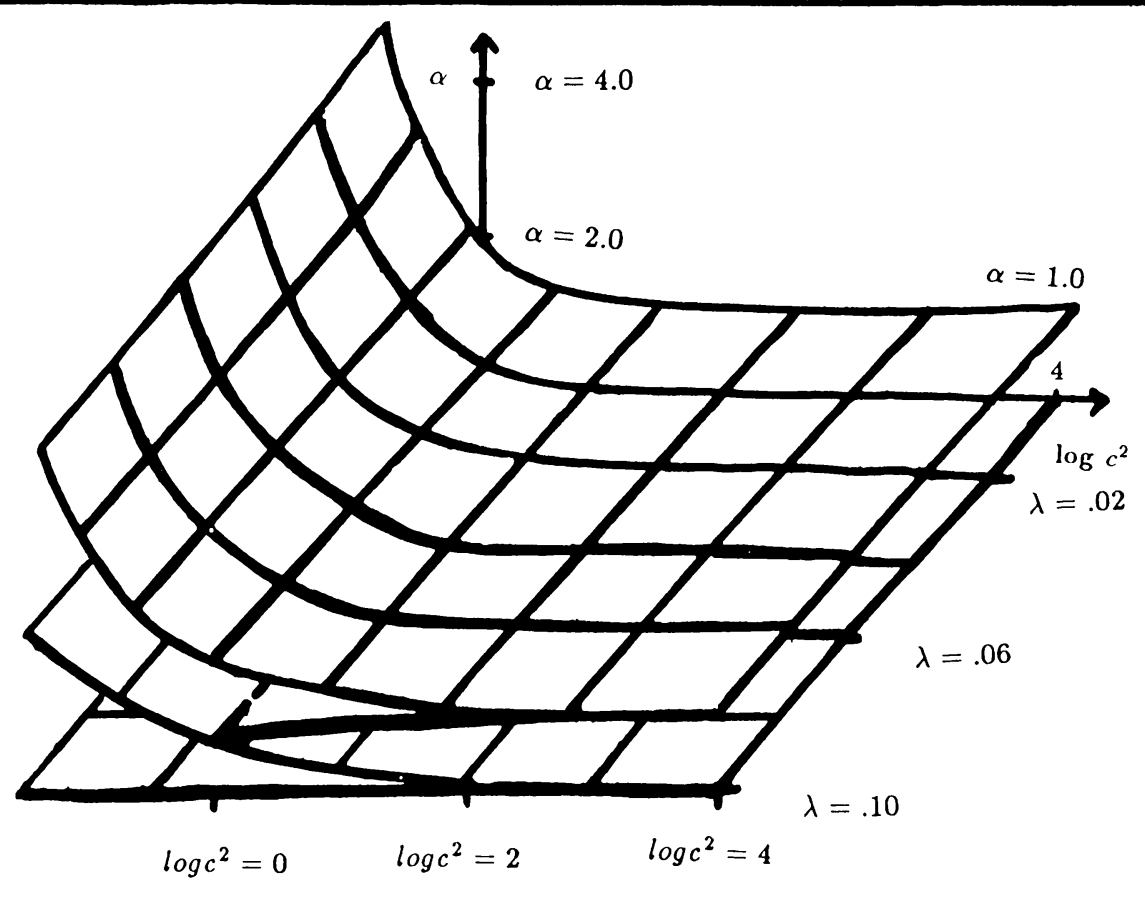

FIG. 8. Parameter values for trajectories which originate at $\mathscr{S}_{-}$and terminate at $\mathscr{S}_{0}$ without oscillations. 
is in the equilibrium state lags behind the point where $\phi=0$ (the point at which the phase transition takes place), resulting in a more spread out front. These general characteristics seem to be those one would expect. However, this model also includes some which are unexpected. We shall discuss these and some of their implications in the next section.

Conclusions. As stated earlier, this phase field model predicts a limit to the amount of supercooling which can be applied if one is to have solutions which are constant in some region of the $\left(x^{\prime}, t^{\prime}\right)$ space. Since this is necessary for the existence of bounded traveling wave solutions of the form we have been considering here, we find the restriction on the supercooling to be

$$
\left[T_{+\infty}\right]_{\max }=\frac{[\tilde{f}(\phi)]_{\max }}{\sigma},
$$

where $\left[T_{+\infty}\right]_{\max }$ represents the maximum amount the liquid can be supercooled (relative to the freezing point) if traveling wave solutions are to exist. For $\left[T_{+\infty}\right]_{\max }<$ $T_{+\infty}<0$, one should be able to observe traveling wave solutions according to this theory. This is very different from the results of the classical Stefan problem, as shall be discussed below.

We must now consider the relevance of the possible solutions described above. We need to consider the importance of the number of singular points. As was discussed above in relation to Fig. 1, there can be one, two, or three real singular points depending on the value of $\lambda$. If $\lambda$ is greater than $\frac{1}{8}$ then there is only the singular point at $(s, \phi, u)=(0,-1,0)$. The form of traveling waves being considered here require trajectories which both originate and terminate at singular points. There are no such traveling wave solutions for $\lambda>\frac{1}{8}$. For $\lambda=\frac{1}{8}$ there are two singular points, the one at $(0,-1,0)$, and one at $(0,0.5,-0.1875)$. These singular points may be either spirals or saddle points. When $0 \leq \lambda<\frac{1}{8}$ there are two singular points besides the one at $(0,-1,0)$, and their natures have been discussed in detail above. The question now arises as to whether this limitation on $\lambda$ (which implies that $\sigma l / 2 \leq \frac{1}{8}$ ) has a physical basis or if it is just an artifact of the model. This limitation implies that as the entropy coefficient $\sigma$ is increased, the ratio of the latent to specific heats must decrease in order to have traveling wave solutions. The physical interpretation of this and the importance of the value $\lambda=\frac{1}{8}$ are questions which still remain to be addressed in future work.

The locations of the singular points depend only on $\lambda$, however the nature of the singular points are determined by all three parameters as we saw above. To better understand the meaning of these singular points we must examine the derivation of the original equations. As described by Caginalp [2], the starting equations we have used here are based on the assumption of a Helmholtz free energy (such as those encountered in the Landau-Ginzberg theory of phase transitions) of the form

$$
F_{T}\{\phi\} \equiv \int d^{N} x\left[\frac{1}{2} \xi^{2}(\nabla \phi)^{2}+\frac{1}{8}\left(\phi^{2}-1\right)^{2}-\sigma T \phi\right],
$$

where the $\left(\phi^{2}-1\right)^{2}$ term is a commonly used prototype double-well potential as mentioned above. This double-well potential indicates that for $\phi= \pm 1$ (which 
correspond to the liquid and solid states, respectively) the free energy will take on its smallest values, indicating the most stable states. For $\phi$ values inbetween these the free energy will not be at its minimum, indicating some form of transition state. In light of this, we see that as $|\phi|$ at any point in the phase space diverges from unity, the state of the material at that point becomes more "transitory" or thermodynamically unstable in nature. While it may seem strange that the "liquid" form of the substance we are dealing with here is not in the most stable form as $X \rightarrow+\infty$, upon closer examination this is seen to be necessary since we are here considering a super-cooled liquid, which certainly is not a stable state. As a result, in the case where there are two singular points in the $\phi>0$ region, there are two possible states for the supercooled liquid. Due to the nature of the potential well being considered here, it seems that the singular point with a $\phi$ value closer to the thermodynamically stable state (which in this case is $\phi=+1$ ) should correspond to transitions which would be easier to observe experimentally. As to whether this should rule out solutions which enter the other singular point is not clear, however it seems that the solutions entering the other singular point would be harder to see experimentally due to their greater instability. The stability of these traveling waves still remains to be addressed and is under consideration.

We can therefore draw the following conclusions from what we have seen so far: as $\lambda$ (which involves the ratio of the latent to the specific heat of the material and the entropy coefficient $\sigma$ ) is increased, the value of $u_{+\infty}$ decreases. If this change in $\lambda$ is due to a change in $\sigma$, then the dimensioned amount of supercooling which must be applied at the boundary for solutions to exist does not change since this is given by $T_{+\infty}=-(\lambda / \sigma)\left(1+\phi_{+\infty}\right)=-(l / 2)\left(1+\phi_{+\infty}\right)$. As $l$ is increased, however, the amount of supercooling necessary to have traveling wave solutions (and thereby the instability of these states) increases until one reaches the limiting value of $\lambda=\frac{1}{8}$, above which there are no solutions of the form being considered here. For any $\lambda$ less than this there are two possible states for the supercooled liquid to be in. Depending on the values of $\lambda$ and $\alpha$ only certain values for the speed $c$ are allowable if solutions are to exist. For any given $(\alpha, \lambda)$ pair one cannot always pick a speed $c$ such that there is a traveling wave solution which is monotonic.

We shall compare the results obtained above with those obtained from a similar traveling wave analysis of the Stefan problem. Here we have

$$
\begin{aligned}
& \left(T_{s}\right)_{t^{*}}=\left(\frac{k}{\rho C}\right)_{s}\left(T_{s}\right)_{x^{*} x^{*}}, \\
& \left(T_{l}\right)_{t^{*}}=\left(\frac{k}{\rho C}\right)_{l}\left(T_{l}\right)_{x^{*} x^{*}},
\end{aligned}
$$

with the normal Stefan condition along the phase boundary

$$
L \frac{d S}{d t^{*}}=\left[-k_{s}\left(T_{s}\right)_{x^{*}}+k_{l}\left(T_{l}\right)_{x^{*}}\right]
$$

where the subscripts $s$ and $l$ refer to the solid and liquid states, respectively, and where $T$ is the temperature, $k$ is the thermal conductivity coefficient, $\rho$ is the density of the material and $C$ is the heat capacity. Assuming that there is no volume 
change during the transition and that thermal diffusivities for the solid and liquid are equal and that the solid is always at the fusion temperature we can rescale the problem using

$$
\begin{gathered}
u=\frac{T_{l}-T_{f}}{\widetilde{T}} ; \quad \tilde{x}=\frac{x^{*}}{\gamma} ; \quad \tilde{t}=\left(\frac{k_{l}}{\rho C \gamma^{2}}\right) t^{*} ; \\
s=\left(\frac{\rho C \gamma}{k_{l}}\right) S,
\end{gathered}
$$

where $\gamma$ and $\widetilde{T}$ are characteristic length and temperature scales, respectively. This leads to

$$
u_{\tilde{t}}=u_{\widetilde{x x}}, \quad \text { (in the liquid region) }
$$

with a condition on the front given by

$$
u_{\tilde{x}}=-\beta \frac{d s}{d \widetilde{t}}, \quad(\text { for } \tilde{x}=s(\widetilde{t}))
$$

where

$$
\beta=L k / \widetilde{T}(\rho C \gamma)^{2} .
$$

If we transform to a moving frame

$$
\begin{gathered}
t=\tilde{t}, \\
X=\tilde{x}-c \tilde{t},
\end{gathered}
$$

and specify that the interface moves with speed $c$ (i.e., $d s / d \tilde{t}=c$ ), we have (for a steady traveling wave)

$$
-c u_{X}=u_{X X} ; \quad \text { (in the liquid region) }
$$

with

$$
u_{X}=-\beta c ; \quad(\text { along the front } X=0) \text {. }
$$

This implies

$$
u=\beta\left(-1+e^{-c X}\right) .
$$

Since we are considering cases where $\beta>0$, we see that the Stefan problem also predicts traveling wave solutions when the liquid is supercooled. In contrast to what was found with the phase field model, the Stefan problem has no restrictions on the speed of the traveling wave. The phase field model, as discussed above, implies that there is at most one speed at which the wave can travel if solutions are to be monotonic (as they are in the Stefan problem). We also see that the Stefan problem predicts that only one value of supercooling is allowed if traveling wave solutions are to exist. The phase field model predicts that such solutions can exist for more than one value of supercooling (though it seems that due to physical reasons one would be more thermodynamically stable). In addition, the phase field model puts limitations on the ratio of the latent and specific heats if these solutions are to exist. Such limitations do not come about as a result of solving the Stefan problem. These differences between the classical Stefan problem and the phase field model are difficulties which must be resolved. We would expect there to be differences in the 
solutions from the two models since it is known that the Stefan formulation does not include all of the physics involved, and the phase field model was developed so that some of these extra physical properties could be examined. The restrictions we are seeing here in the speed might then be due to actual physical properties of the material which have been included in this model. While it seems likely that there should be some restrictions on the speed of the traveling wave, there is no basis at this time from which to conclude that the restrictions found here are the correct ones. Similarly, one could question whether physically there should be only one temperature for which traveling wave solutions exist, of if there should be multiple possibilities. An analysis of the validity of these restrictions based on physically realizable systems yet remains to be examined. We should mention that it has been shown [10] that the phase field model does reduce down to the Stefan problem in a singular limit. This demonstrates that the model does give the classical results for some limit, but the differences reported in this work still need to be addressed to determine if its behavior is correct outside of this special case.

The use of these traveling wave solutions to at least in part evaluate the nature of this phase field model should be valid since we would expect that they are an asymptotic representation of the solution to the overall problem as one approaches the front. This can rather easily be proven for the Stefan problem where it is indeed found that the solution given above for the traveling wave in the Stefan problem is the first term in an asymptotic expansion of the solution to the full problem near the front [11]. We would expect that this would also hold for the phase field model, thus allowing for the results found here to give further insight into the nature of the solutions resulting from this model.

It has been seen that the use of this model results in some very stringent restrictions on the ratio of the latent to specific heats. We have also seen that the results are also dependent on the form of the potential well chosen to describe the phase change. As was seen above, if $g(\phi)$ represents the potential well, then $\left[g\left(\phi^{\prime}\right)\right]_{\max } / \sigma$ gives that maximum amount of supercooling which can be used if traveling wave solutions are to exist. It was also found that there is a restriction of the form $l \sigma / 2 \leq \frac{1}{8}$ if traveling wave solutions are to exist. In addition, we found that there was at most one speed at which the front can move with this type of solution (if we wish to only consider solutions which enter the more stable critical point). Future work in the development of this model must then address the evaluation of these restrictions: first, whether they are due to the physics of the system involved. If so, then second, does this model give the correct qualitative and/or quantitative description of the ranges of parameters for which solutions exist as well as the nature of the solutions themselves. As mentioned above, the stability of these traveling waves also remains to be studied.

Acknowledgments. The author would like to gratefully acknowledge the help and support of Julian D. Cole. 


\section{REFERENCES}

[1] G. Caginalp, Surface tension and supercooling in solidification theory, (Proc. Sitges conference on statistical mechanics, Sitges, 1984) ed. by L. Garrido, Lecture Notes in Physics, Vol. 216, Springer, New York, 1984

[2] G. Caginalp, An analysis of a phase field model of a free boundary, Arch. Rational Mech. Anal. 92, 205-245 (1986)

[3] G. Caginalp and P. C. Fife, Phase field methods for interfacial boundaries, Phys. Rev. B. 33, 7792-7794 (1986)

[4] G. Caginalp and P. C. Fife, Higher-order phase field models and detailed anisotropy, Phys. Rev. B. 34, 4940-4943

[5] G. Caginalp and P. C. Fife, Qualitative properites of solutions, Non-Linear Parabolic equations, Pitman, Boston, 1987

[6] G. Caginalp and P. C. Fife, Dynamics of layered interfaces arising from phase boundaries, SIAM J. Appl. Math. 48 506-157 (1988)

[7] G. Caginalp and B. McLeod, The internal transition layer for an ordinary differential equation arising from solidification theory, Quart. Appl. Math. 44, 155-168 (1986)

[8] J. T. Lin, The numerical analysis of a phase field model in moving boundary problems, SIAM J. Numer. Anal. 25, 1015-1031 (1988)

[9] L. I. Rubinstein, The Stefan Problem, Amer. Math. Soc. Transl., vol. 27, Amer. Math. Soc., Providence, RI, 1971

[10] G. Caginalp, Stefan and Hele Shaw Type Models as Asymptotic Limits of the Phase Field Model, in press

[11] J. W. Wilder, An Asymptotic Solution to the Stefan Problem Near the Interface, unpublished work 